\title{
Transcatheter Intra-Arterial Infusion Combined with Interventional Photothermal Therapy for the Treatment of Hepatocellular Carcinoma
}

This article was published in the following Dove Press journal: International Journal of Nanomedicine

\author{
Jun Zhou' \\ Gonghao Ling' \\ Jia Cao' \\ Xun Ding' \\ Xingnan Liao' \\ Meng Wu (D) $^{2}$ \\ Xinyu Zhou ${ }^{3}$ \\ Haibo Xu (D) \\ QingYun Long'
}

'Department of Radiology, Zhongnan Hospital of Wuhan University, Wuhan 43007I, Hubei, People's Republic of China; ${ }^{2}$ Department of Ultrasound, Zhongnan Hospital of Wuhan University, Wuhan 43007I, Hubei, People's Republic of China; ${ }^{3}$ Department of Radiation and Medical Oncology, Zhongnan Hospital of Wuhan University, Wuhan 43007I, Hubei, People's Republic of China
Correspondence: Haibo Xu;

QingYun Long

Department of Radiology, Zhongnan

Hospital of Wuhan University, No. 169

Donghu Road, Wuhan 43007I, Hubei,

People's Republic of China

Tel +86 I35450094I6; +86 I5827339526

Email xuhaibol I20@hotmail.com;

long9y2005@sina.com
Background: Photothermal therapy (PTT) has great potential application in the treatment of tumors. However, due to the low penetration of near-infrared light (NIR) and the low concentration of nanomaterials in the tumor site, the application of PTT has been limited.

Purpose: The objective of this study was to investigate the therapeutic effect of transcatheter intra-arterial infusion of lecithin-modified Bi nanoparticles (Bi-Ln NPs) combined with interventional PTT (IPTT) on hepatocellular carcinoma.

Methods: Bi-Ln NPs were prepared by emulsifying the hydrophobic Bi nanoparticles and lecithin, and the photothermal conversion and cytotoxicity of Bi-Ln NPs were then measured by infrared imaging and MTT (3-(4,5-dimethylthiazol-2-yl)-2,5-diphenyltetrazolium bromide) assay, respectively. Twenty-four VX2 hepatic carcinoma rabbits were randomly divided into four groups. Rabbits in group A received Bi-Ln NPs by intra-arterial infusion and NIR laser treatment (IA Bi-Ln NPs + Laser), group B received Bi-Ln NPs by intravenous infusion and NIR laser treatment (IV BiLn NPs + Laser), group C received PBS (phosphate buffer saline) via intra-arterial infusion with NIR laser treatment (IA PBS + Laser), group D received PBS via intra-arterial infusion (IA PBS). Transcatheter intra-arterial infusion was conducted by superselective intubation under digital subtraction angiography (DSA) guidance. IPTT was performed by introducing an NIR optical fiber access to the rabbit VX2 hepatic carcinoma under real-time ultrasound guidance. Magnetic resonance imaging (MRI) was performed to evaluate the tumor size. Hematoxylin and eosin (H\&E) stain and terminal deoxynucleotidyl transferase dUTP nick end labeling (TUNEL) were conducted 7 days after treatment to evaluate the necrosis rate and viability of tumor, respectively. Results: The Bi-Ln NPs have the advantages of good biological compatibility and high photothermal conversion efficiency. Minimally invasive transcatheter intra-arterial infusion can markedly increase the concentration of Bi-Ln NPs in tumor tissues. IPTT can contribute to the significant improvement in the photothermal efficiency of Bi-Ln NPs. Compared to other groups, the group of IA Bi-Ln NPs + Laser showed a significantly higher tumor inhibition rate (TIR) of $93.38 \pm 19.57 \%$, a higher tumor necrosis rate of $83.12 \pm 8.02 \%$, and a higher apoptosis rate of $(43.26 \pm 10.65 \%)$ after treatment.

Conclusion: Transcatheter intra-arterial infusion combined with interventional PTT (IPTT) is safe and effective in eradicating tumor cells and inhibiting tumor growth and may provide a novel and valuable choice for the treatment of hepatocellular carcinoma in the future.

Keywords: transcatheter intra-arterial infusion, interventional photothermal therapy, Bi nanoparticles, hepatocellular carcinoma

\section{Introduction}

Hepatocellular carcinoma (HCC) represents $75-90 \%$ of primary liver malignant tumors and is a major cause of tumor-related mortality worldwide. ${ }^{1}$ Statistically, the 
cancer survival rate can be significantly improved if it is diagnosed at early stages. ${ }^{2}$ However, only $25 \%$ of the total HCC patients are diagnosed within the early stage. ${ }^{3,4}$

Currently, surgical therapies, liver transplantation, tumor ablation, transhepatic arterial chemoembolization (TACE), radiotherapy and systemic therapies are the main therapeutic modalities for HCC. Unfortunately, more than $80 \%$ of patients have already lost the opportunity for surgical removal of tumors by the time of diagnosis, ${ }^{5}$ and liver transplantation is limited by high cost, donor shortage, and poor prognosis. ${ }^{6-10}$ The therapeutic effect of PEI and RFA will be compromised when tumors are larger than $3.0 \mathrm{~cm}$, and the rate of tumor recurrence following RFA or PEI is high. ${ }^{11,12}$ TACE and radioembolization were developed to carry chemoembolization agents and radioactive isotopes to the lesion through the hepatic artery, ${ }^{13,14}$ the reduced arterial inflow caused by injected embolic agents can reduce the supply of oxygen and glucose for tumor metabolism and, therefore, can increase cell death and the therapeutic effect. ${ }^{15,16}$ However, the issue of high recurrence rates and the potential exposure of radioactive isotopes for other organs should be given significant consideration.

PTT commonly relies on photothermal conversion agents (PTAs) to generate sufficient heat under NIR light irradiation for tumor therapy. ${ }^{17-19}$ Recent studies have shown that bismuth nanoparticles can serve as CT contrast agents and PTAs due to their good performance. ${ }^{20-25} \mathrm{Bi}$ is the highest atomic number metal element among the "nonradioactive elements" and possesses an ultrahigh X-ray attenuation coefficient. $^{26,27}$ Moreover, Bi shows good biocompatibility and low toxicity. ${ }^{28,29}$ Importantly, Bi possesses a high NIR absorption coefficient. ${ }^{21,22,25}$

PTT has attracted extensive attention because of certain advantages, including minimal invasiveness, reduced side effects, and high specificity. ${ }^{30,31}$ However, PTT is restricted by several limitations, including a limited light penetration depth for deeply buried tumors, the possibility of damaging normal tissues, compromised therapeutic effects by threshold laser power for skin tolerance, ${ }^{32}$ and low concentrations of nanomaterials in tumors by intravenous administration. ${ }^{33}$ Given these issues, the transcatheter intra-arterial infusion has been applied in previous studies, ${ }^{34-36}$ but studies have seldom evaluated the efficacy of transcatheter intra-arterial infusion combined with IPTT compared with other therapy modalities for hepatic carcinoma. Herein, to improve the therapeutic effect of HCC, we developed a new therapeutic method of transcatheter intra-arterial infusion of Bi-based nanoparticles (Bi-Ln NPs) combined with IPTT. Digital subtraction angiography (DSA)-guided transcatheter intra-arterial infusion and real-time ultrasound-guided IPTT are used to overcome the low concentrations of nanomaterials in the lesions and the limited penetration of NIR, respectively. Therefore, the superior photothermal property of Bi-Ln NPs can more effectively eliminate hepatic carcinoma. The detailed therapeutic route is presented in Scheme 1.

\section{Materials and Methods}

\section{Materials}

$\mathrm{Bi}(\mathrm{NO} 3) 3 \cdot 5 \mathrm{H} 2 \mathrm{O}$ and oleylamine were purchased from Sigma Aldrich (St Louis, MO, USA). 1-Dodecanethiol was purchased from Innochem Technology Co., Ltd (Beijing, People's Republic of China). Ethanol and cyclohexane were purchased from Sinopharm Chemical Reagent Co., Ltd. (Beijing, People's Republic of China). Trypsin-EDTA $\left(\right.$ Gibco $\left.^{\circledR}\right)$, dulbecco's modified eagle medium (DMEM, HyClone ${ }^{\circledR}$ ), fetal bovine serum (FBS, Gibco ${ }^{\circledR}$ ), and penicillin-streptomycin solution (HyClone ${ }^{\circledR}$ ) were provided by Beijing Dingguo Changsheng Biotechnology Co., Ltd. (Beijing, People's Republic of China). MTT was purchased from Sigma Aldrich (St Louis, MO, USA). All chemicals were of analytical grade.

\section{Synthesis of Hydrophobic $\mathrm{Bi}$ Nanoparticles (Bi NPs)}

Bi NPs were synthesized by modification of a reported method. ${ }^{29}$ Under an $\mathrm{N}_{2}$ atmosphere, $\mathrm{Bi}\left(\mathrm{NO}_{3}\right)_{3} \cdot 5 \mathrm{H}_{2} \mathrm{O}(2.0 \mathrm{~g})$, oleylamine $(2 \mathrm{~mL})$ and 1-dodecanethiol $(8 \mathrm{~mL})$ were mixed in a three-neck flask. After purging for $1 \mathrm{hr}$ at $100^{\circ} \mathrm{C}$, the mixture solution was further heated and stained at $180^{\circ} \mathrm{C}$ for 30 mins and cooled to room temperature naturally. To obtain purified Bi nanoparticles, the mixture solution was washed with ethanol $(20 \mathrm{~mL})$ three times and collected by centrifugation at $8000 \mathrm{rpm}$. The collected Bi NPs were dispersed in cyclohexane for further experiments.

\section{Preparation of Bi-Ln NPs}

The Bi-Ln NPs were prepared by emulsification methods. In brief, Bi NPs (10 mg) and lecithin (100 mg) were dispersed in $3 \mathrm{~mL}$ of DCM (dichloromethane)/cyclohexane $(2 / 1, V / V)$ with stirring. After sonicating for $30 \mathrm{~min}$, $20 \mathrm{~mL}$ of ultrapure water was added to obtain the emulsion. After that, the organic solvents were removed under reduced pressure. Subsequently, the water-dispersed 

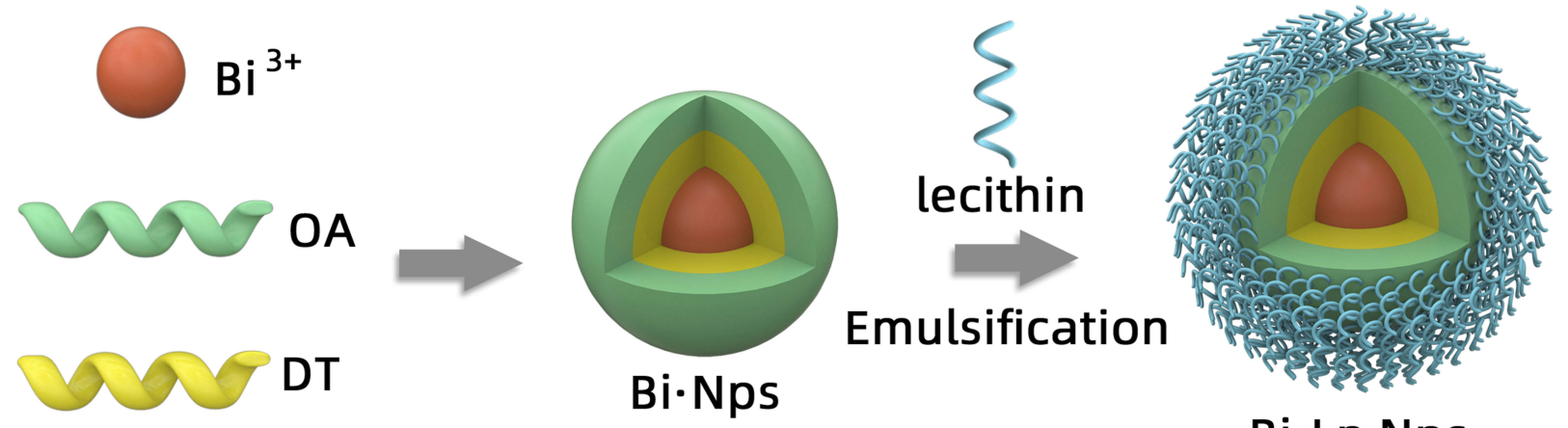

\section{$\mathrm{Bi} \cdot \operatorname{Ln} \mathrm{Nps}$}

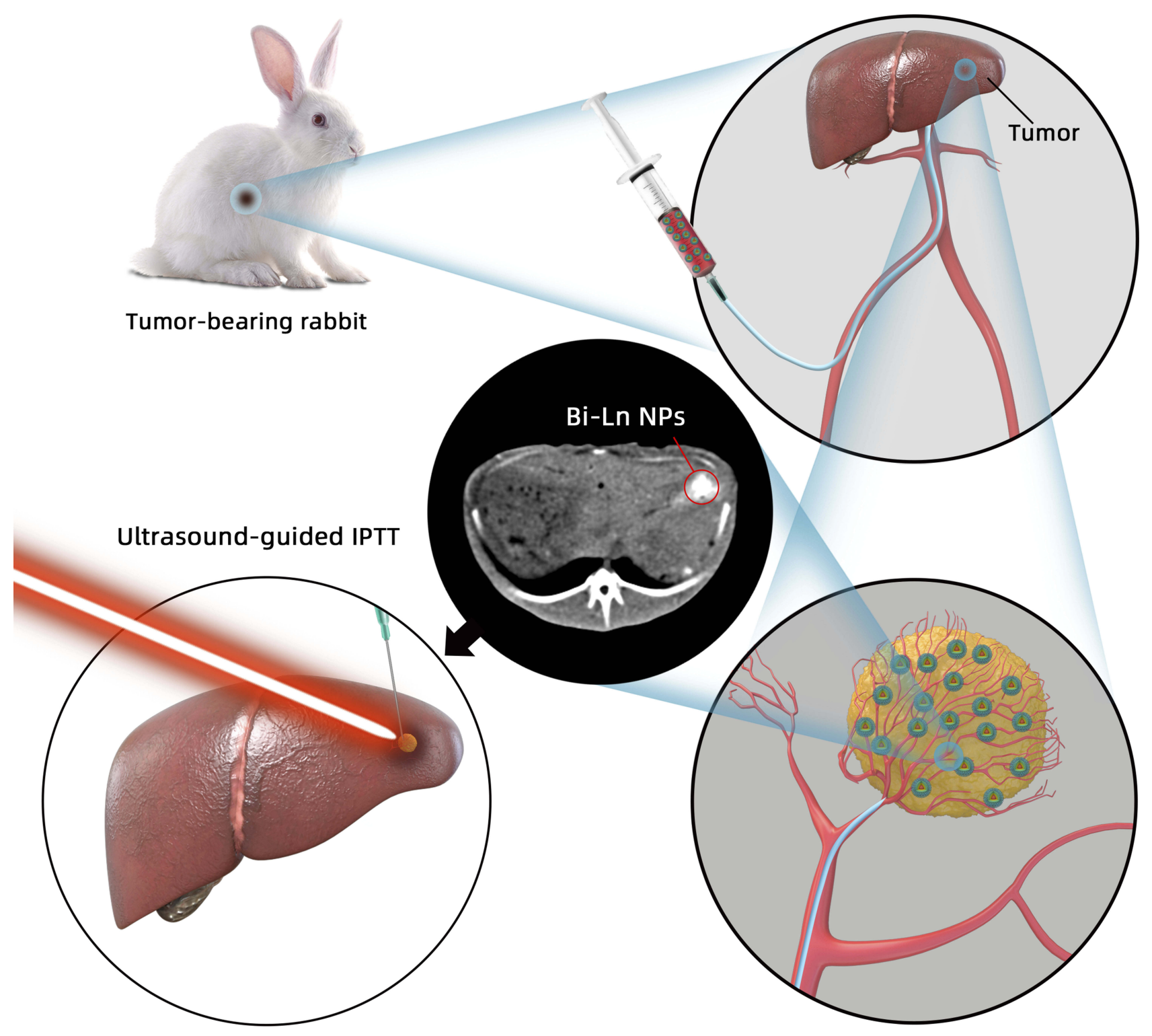

Scheme I Schematic illustration of transcatheter intra-arterial infusion combination with IPTT for the treatment of rabbit VX2 hepatic Carcinoma.

solution was centrifuged at $5000 \mathrm{rpm}$ for $10 \mathrm{~min}$ to eliminate redundant lecithin. The obtained Bi-Ln NPs were dispersed in water and stored at $4^{\circ} \mathrm{C}$ for further study.
The Bi-Ln NPs loading capacity was estimated by ICPAES (Inductively Coupled Plasma-Atomic Emission Spectrometry). 


\section{Characterization of the Bi-Ln NPs}

Dynamic light scattering (DLS) was performed with Zetasizer Nano ZS (Malvern Instruments Ltd, UK) to determine the average hydrodynamic diameter and polydispersity index (PDI) of Bi-Ln NPs. A transmission electron microscopy system (TEM, JEOL JEM-100CXII, 100 kV, Japan) was used to measure the morphologies of Bi-Ln NPs. The UV-vis-NIR absorption spectra were collected using a Hitachi U-3900 spectrophotometer. X-ray diffraction (XRD) spectra were characterized on a PANalytical/X'Pert Pro X-ray diffractometer (PANalytical B.V., Nederland). Stability of Bi-Ln NPs were measured by DSL to monitor the absorption peaks of Bi-Ln NPs during incubation in PBS for 7 days. Cycle heating experiments ( 3 mins of irradiation followed by a 5 -mins cooling period) were carried out to test photostability properties under repeated NIR light irradiation $\left(808 \mathrm{~nm}, 1.0 \mathrm{~W} / \mathrm{cm}^{2}\right)$.

\section{Animal Model}

Twenty-four Japanese big-ear rabbits, male and female, were purchased from Wuhan Wanqianjiaxing Biotechnology Co., Ltd. (Wuhan, People's Republic of China). All rabbits were acclimatized for 2 weeks under standardized laboratory conditions, including a temperature-controlled room with a $12 \mathrm{hrs} \mathrm{light/dark} \mathrm{cycle,} \mathrm{single} \mathrm{cages,} \mathrm{standard} \mathrm{laboratory}$ diet and drinking water ad libitum. The rabbits had a mean weight of $2915 \pm 492$ g. The Institutional Animal Ethics Committee of Wuhan University approved the study protocol. All animal studies were performed according to China's National Animal Law on the use of laboratory animals and the institutionally approved protocols of Wuhan University. All experiments were designed to minimize the animals' suffering.

\section{Preparation of the VX2 Hepatic Carcinoma Model}

VX2 tumor tissues were taken from VX2 tumor-bearing rabbits (provided by the Laboratory Animal Research Center, Tongji Medical College of Huazhong University of Science and Technology, People's Republic of China), and tumors were minced into 1 - to $2-\mathrm{mm}^{3}$ pieces in physiological saline. Three percent sodium pentobarbital $(1 \mathrm{~mL} / \mathrm{kg})$ was slowly injected through the auricular vein to anesthetize the rabbit, and a mini-laparotomy incision was made in the upper abdomen. The liver was exposed after carefully dividing the peritoneum. An approximately 1- to $2-\mathrm{mm}^{3}$ VX2 carcinoma was directly implanted into the left lobe of the liver. Finally, the abdominal incision was closed. Subsequently, the tumors were allowed to grow in the rabbits' livers for 2 weeks, and when the tumors were examined to be $10-20 \mathrm{~mm}$ in diameter on the MR scan, the rabbits were used for subsequent experiments.

\section{In vitro and in vivo $\mathrm{CT}$ Imaging}

The CT properties of Bi-Ln NPs were determined by clinical X-ray CT (Definition AS, Siemens, Germany). For in vitro CT contrast efficiency evaluations, various concentrations (16.05, $12.13,9.13,6.81,4.24,3.15,2.66,0.98 \mathrm{mg} / \mathrm{mL}$ ) of Bi-Ln NPs were prepared to obtain the $\mathrm{CT}$ value and the corresponding map. Before measuring the CT values of Bi-Ln NPs in vivo, transcatheter intra-arterial infusion of Bi-Ln NPs was conducted. After 2 weeks of tumor implantation, the intracatheter infusion was performed under a DSA device (Allura, Xper, FD20, Philips Healthcare and Nederland). Tumor-bearing rabbits were anesthetized using 3\% sodium pentobarbital $(1 \mathrm{~mL} / \mathrm{kg})$. After routine disinfection and preparation, an incision was performed to access the rabbit's right femoral artery, the artery was punctured with a 4-F vascular sheath, and a 4-F catheter was used to reach the common celiac artery under DSA guidance. Subsequently, the contrast agent (Iodixanol Injection, Jiangsu Hengrui Medicine Co., Ltd. Jiangsu, People's Republic of China) was injected to confirm the hepatic arteries, and a 2.7-F microcatheter (Progreat, Terumo, Japan) was advanced to reach the left hepatic artery. Then, the tumor location and tumor feeding artery were identified with a hepatic arteriogram. Bi-Ln NPs $(4 \mathrm{mg} / \mathrm{mL}, 3 \mathrm{~mL})$ and PBS ( $3 \mathrm{~mL})$ were successfully injected through the microcatheter located within the tumor feeding artery, respectively. Finally, the catheter was pulled out, the femoral artery was ligated, and the incision was closed. In addition, Bi-Ln NPs $(4 \mathrm{mg} / \mathrm{mL}, 3 \mathrm{~mL}$ ) were intravenously administered into the tumor-bearing rabbits. After infusion of Bi-Ln NPs or PBS, in vivo imaging was obtained with clinical X-ray CT (Definition AS, Siemens, Germany) at $5 \mathrm{~min}, 30 \mathrm{~min}, 1 \mathrm{~h}$, $2 \mathrm{~h}$, and $4 \mathrm{~h}$ after injection with the following parameters: 80 $\mathrm{kV}, 100 \mathrm{~mA}$, and slice thickness $1.0 \mathrm{~mm}$.

\section{In vitro and in vivo Photothermal Conversion of $\mathrm{Bi}-\mathrm{Ln}$ NPs}

The photothermal properties of Bi-Ln NPs were measured in vitro and in vivo by an infrared imaging system. For in vitro study, different concentrations $(0,0.25,0.5,1$, $2 \mathrm{mg} / \mathrm{mL}$ ) of monodispersed Bi-Ln NPs in quartz cuvettes were illuminated with NIR $(808 \mathrm{~nm})$ for $5 \mathrm{~min}$, and the 
temperature was recorded at different time intervals $(0,1,2$, 3, 4, $5 \mathrm{~min}$ ) by a noncontact infrared thermal imager.

For in vivo photothermal conversion of Bi-Ln NPs, BiLn NPs (4 mg/mL, $3 \mathrm{~mL})$ and PBS $(3 \mathrm{~mL})$ were injected through the microcatheter located within the tumor feeding artery, respectively. Five minutes after intra-arterial infusion, the IPTT was performed under real-time ultrasound guidance with an NIR optical fiber $(0.8 \mathrm{~mm}$; Hangzhou Newdoon Technology Co., Ltd, Hangzhou, People's Republic of China) using an 18-gauge $(\mathrm{G})$ percutaneous transhepatic cholangiography (PTC) needle to access the liver tumor, which was then treated with 808-nm laser irradiation at a power density of $1.0 \mathrm{~W} / \mathrm{cm}^{2}$ for $3 \mathrm{mins}$, and the temperature of the tumor tissue was recorded by an infrared thermal imaging system at different time intervals $(0,0.5,1,1.5,2,2.5$, and 3 mins $)$. Meanwhile, the Bi-Ln NPs $(4 \mathrm{mg} / \mathrm{mL}, 3 \mathrm{~mL})$ were intravenously administered into the tumor-bearing rabbits, $1 \mathrm{hr}$ after injection, the tumor was treated with IPTT as mentioned above, and the temperature of the tumor tissue was recorded by the same scheme.

\section{In vitro Photothermal Cytotoxicity of Bi-Ln NPs}

The cytotoxicity of Bi-Ln NPs was determined by the MTT assay. Established hepatic tumor cells (HepG2, ATCC, Manassas, VA, USA) were plated in 96-well plates with 5000 cells/well in a cell incubator for $24 \mathrm{hrs}$. Subsequently, different concentrations $(0.78,1.56,3.13,6.25,12.5,25,50$, $100 \mu \mathrm{g} / \mathrm{mL}$ ) of Bi-Ln NPs were incubated with cells for another $24 \mathrm{hrs}$. After the medium was removed, the cells were washed with PBS three times to discard the unobservable Bi-Ln NPs. After adding fresh medium, cells were treated without or with NIR $\left(808 \mathrm{~nm}, 1 \mathrm{~W} / \mathrm{cm}^{2}\right)$ for $3 \mathrm{~min}$. After further incubation for $24 \mathrm{~h}$, MTT stock solution was added to each well. After further incubation for $4 \mathrm{~h}$, the MTT medium was removed. The formazan blue crystal in each was dissolved by the addition of $150 \mu \mathrm{L}$ of DMSO (dimethyl sulfoxide), and the absorbance of the solution was measured using a microplate reader at $570 \mathrm{~nm}$.

\section{In vivo IPTT}

For IPTT, the tumor-bearing rabbits were randomly divided into four groups ( $n=6$ per group). Rabbits received Bi-Ln NPs ( $4 \mathrm{mg} / \mathrm{mL}, 3 \mathrm{~mL}$ ) separately by intra-arterial and intravenous infusion. Rabbits in group A (IA Bi-Ln NPs+Laser) received $\mathrm{Bi}-\mathrm{Ln}$ NPs by intra-arterial infusion with NIR laser treatment, rabbits in group B (IV Bi-Ln NPs+ Laser) received Bi-Ln NPs by intravenous infusion with NIR laser treatment, rabbits in group $\mathrm{C}$ received $\mathrm{PBS}$ via intra-arterial infusion with NIR laser treatment (IA PBS+Laser), and rabbits in group D received PBS via intra-arterial infusion (IA PBS). Five minutes after infusion in groups $\mathrm{A}$ and $\mathrm{C}$ and. One hour after infusion in group B, VX2 hepatic carcinoma was treated with IPTT $\left(808 \mathrm{~nm}, 1.0 \mathrm{~W} / \mathrm{cm}^{2}\right)$ for 3 mins. Subsequently, the tumor size was measured by magnetic resonance imaging (MRI) at baseline and at 1, 3, and 7 days after treatment. MRI examination was performed with a $3.0 \mathrm{~T}$ MR scanner (Prisma, Siemens, Erlangen, Germany) with a human knee coil. A BLADE TSE T2-weighted image (T2WI) sequence was acquired with the following parameters: $\mathrm{TR}=2300 \mathrm{~ms}$, $\mathrm{TE}=104 \mathrm{~ms}$, section thickness $=3 \mathrm{~mm}$, and voxel size $=0.6$ $\times 0.6 \times 3.0 \mathrm{~mm}^{3}$.

The tumor volume was evaluated in T2WI according to the following equation: $\mathrm{V}=0.5 \times \mathrm{d} 1 \times \mathrm{d} 2^{2}(\mathrm{~d} 1=$ maximum diameter of the tumor, $\mathrm{d} 2=$ minimum diameter perpendicular to $\mathrm{d} 1)$. The tumor growth ratio $=\mathrm{V} / \mathrm{V} 0(\mathrm{~V} 0$ and $\mathrm{V}$ are initial and final tumor volumes). The tumor inhibition rate (TIR) was calculated based on the following equation: TIR $=[1-(\mathrm{Vt}-\mathrm{Vt} 0)$ $/(\mathrm{Vp}-\mathrm{Vp} 0)] \times 100 \%(\mathrm{Vt}$ and $\mathrm{Vt} 0$ represent the final and initial tumor volumes of the treatment group, respectively; $\mathrm{Vp}$ and $\mathrm{Vp} 0$ represent the final and initial tumor volumes of the PBS group, respectively).

\section{Histological Examination}

All tumor-bearing rabbits were euthanized with an intravenous injection of a lethal amount $(60 \mathrm{mg} / \mathrm{kg})$ of sodium pentobarbital under deep anesthesia after the last MRI test on day 7. The tumors were carefully dissected from the liver, and each tumor was fixed in $10 \%$ buffered formalin. The specimen was then embedded in paraffin and cut into $4-\mu \mathrm{m}$ sections. Thereafter, representative tissue slides were stained with hematoxylin for 5 mins followed by staining with eosin for 3 mins for general inspection. Subsequently, the viability in the tumor tissue was assessed by means of a TUNEL assay kit (Roche, Shanghai, People's Republic of China). The obtained slides were observed and imaged with an optical microscope (OLYMPUS IX51, Japan). TUNEL-positive cells (apoptotic cells) were evaluated using Image $\mathrm{J}$ analysis software (version 1.8.0, National Institutes of Health, Bethesda, MD, USA). The rate of apoptotic cell nuclei is defined as apoptotic positive cell nuclei/total cell nuclei in the field. The slides were examined and analyzed by a pathologist who was blinded to the treatment. 


\section{Statistical Analysis}

All data are expressed as the mean \pm SD. Group differences were determined using a one-way analysis of variance (ANOVA). If the ANOVA test found significant differences among groups, a post hoc $t$-test was performed to assess the intergroup differences with LSD correction for multiple comparisons. P values less than 0.05 were considered significantly different. All data analyses and graphical representations were performed and generated in Statistical Package for the Social Sciences version 16.0 (SPSS, Inc., an IBM Company, Chicago, IL) and GraphPad Prism 7.0 (GraphPad Software Inc., San Diego, CA).

\section{Result and Discussion}

\section{Preparation and Characterization of Bi-Ln NPs}

In this study, the "one-pot" method was used to synthesize hydrophobic Bi microparticles based on oleylamine and 1-dodecanethiol coated on Bi microparticles to stabilize the Bi NPs and to prevent aggregation of Bi NPs in organic solution. The average size of Bi NPs determined by DLS was approximately $10 \mathrm{~nm}$ in cyclohexane. TEM was used to exhibit Bi NPs with a well-defined spherical shape (Figure 1A). To endow Bi NPs with water dispensability and good histocompatibility, lecithin was utilized to modify the surface of Bi NPs by ultrasonic emulsification. After modification with lecithin, the TEM imaging of the Bi-Ln NPs revealed a smooth surface (Figure 1B), demonstrating successful lecithin decoration. As depicted in Figure 1C, Bi-Ln NPs exhibited a uniform size with a diameter of approximately 200 $\mathrm{nm}$ and good dispersion (PDI $=0.102)$ in water, making them suitable for biological systems. Bi-Ln NPs showed strong absorption around NIR (700-900 nm) (Figure 1D), demonstrating that Bi-Ln NPs possessed a potential for NIR photothermal conversion. As depicted in Figure 1E, the absorption peaks of Bi-Ln NPs remained constantly during incubation in PBS for 7 days, suggesting excellent stability. As shown in Figure $1 \mathrm{~F}$, the XRD pattern of Bi-Ln NPs demonstrated a rhombohedral structure through the intense peaks. Furthermore, cycle heating experiments showed that Bi-Ln NPs have good photostability properties under repeated NIR light irradiation ( $808 \mathrm{~nm}, 1.0 \mathrm{~W} / \mathrm{cm}^{2}$ ) (Figure 1G), which was crucial for the following applications of PTT.

\section{DSA Imaging}

The VX2 tumor is derived from a papilloma virus-induced rabbit epithelial cell line. Rabbit hepatic VX2 carcinoma is a rapidly growing, hypervascular tumor that is mainly vascularized by the hepatic artery, similar to human hepatocellular carcinoma; thus, the hepatic VX2 carcinoma model has been increasingly used in recent years as a model for both HCC and interventional therapies. ${ }^{37-39}$ In this study, after 2 weeks of tumor implantation, a superselective hepatic arteriogram showed obvious staining of the tumor in the left lobe of the liver and a rich blood supply (Figure 2A and C). After the injection of BiLn NPs (Figure 2B), X-ray fluoroscopic images showed obvious Bi-Ln NPs aggregation in the tumor area. However, there were no significant density changes in the tumor area after injection of PBS (Figure 2D). This difference in density was later confirmed by $\mathrm{CT}$ imaging.

\section{In vitro and in vivo $C T$ Imaging}

CT imaging technology, with its operability and excellent depth penetration, has been widely used for biomedical imaging. To improve the diagnostic accuracy and to display the rate of lesions, CT contrast agents are commonly applied to effectively enhance scanning to distinguish lesion tissue from biological systems with differential X-ray absorption features. Compared to frequently used I-, Pt-, Au-based nanomaterials, Bi-based nanomaterials possess larger X-ray absorption coefficients (Bi $5.74 \mathrm{~cm}^{2} \mathrm{~kg}-1$ at $100 \mathrm{keV}$ ), ${ }^{23}$ and an increasing number of Bi-based nanomaterials have been used as CT contrast agents. ${ }^{28}$

For CT imaging in vitro, a series concentration of BiLn NPs was prepared, and CT images were obtained. As depicted in Figure 3A, CT images exhibited greater brightness with increasing concentrations of Bi-Ln NPs, similar to the $\mathrm{CT}$ values (Figure 3B). These phenomena were attributed to the large atomic number and high X-ray attenuation coefficient of bismuth.

For CT imaging in vivo, CT examination was carried out on tumor-bearing rabbits after the intra-arterial (group A) and intravenous (group B) administrations of Bi-Ln NPs and the intra-arterial infusion of PBS (group C), respectively. As depicted in Figure $3 \mathrm{C}$ and $\mathrm{D}$, in group $\mathrm{A}$, the $\mathrm{CT}$ values and $\mathrm{CT}$ signals of the tumor site increased significantly compared to other groups. Five minutes after infusion, the $\mathrm{CT}$ values increased from $39.9 \mathrm{HU}$ to $194.2 \mathrm{HU}$ and reached a maximum. For group $\mathrm{B}, 1 \mathrm{hr}$ after infusion, the CT values increased from $40.5 \mathrm{HU}$ to $63.7 \mathrm{HU}$ and reached a maximum. The $\mathrm{CT}$ values showed no significant increase in group $\mathrm{C}$.

In most current studies, PTAs are usually administered by intravenous injection, the tumor accumulation of PTAs 

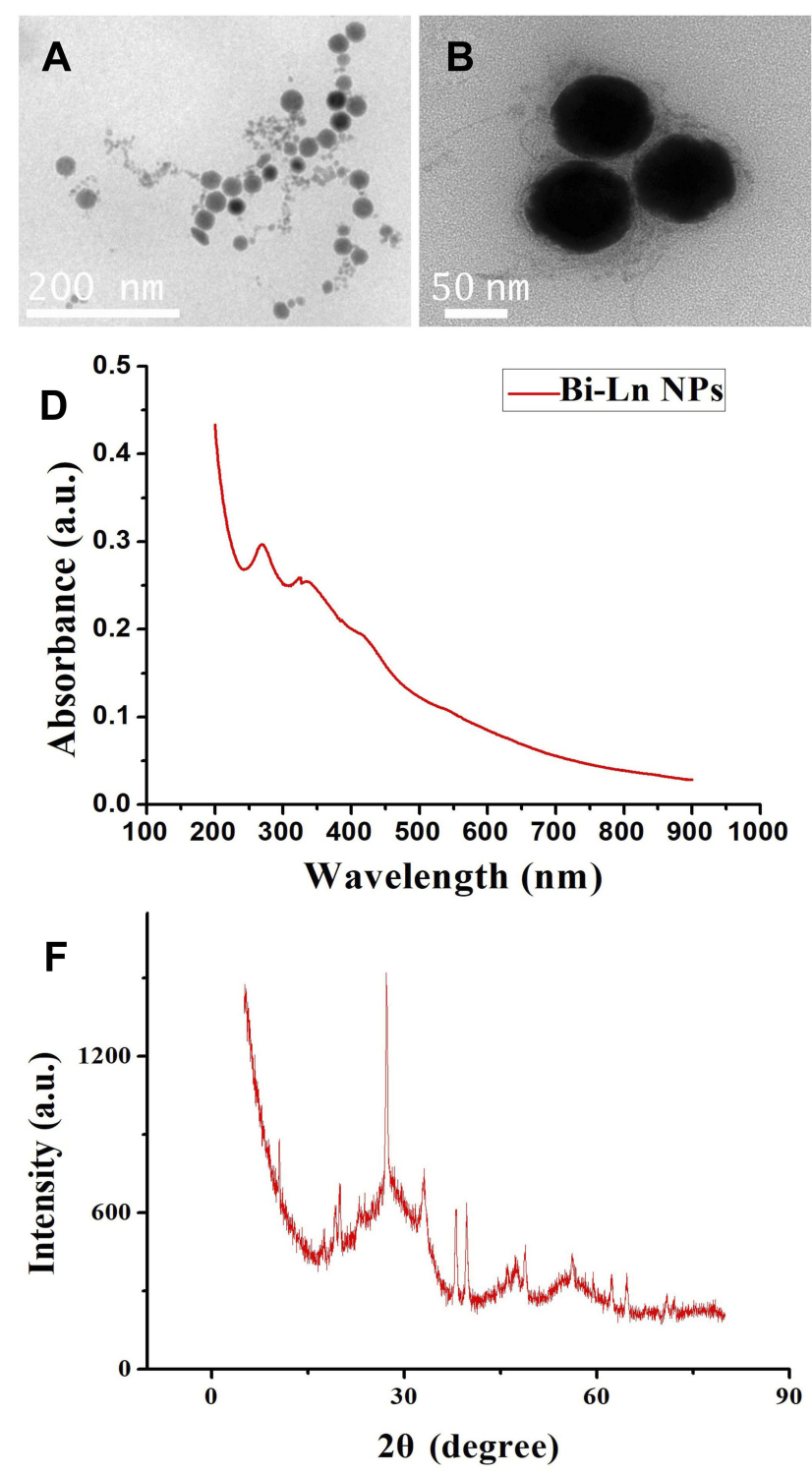
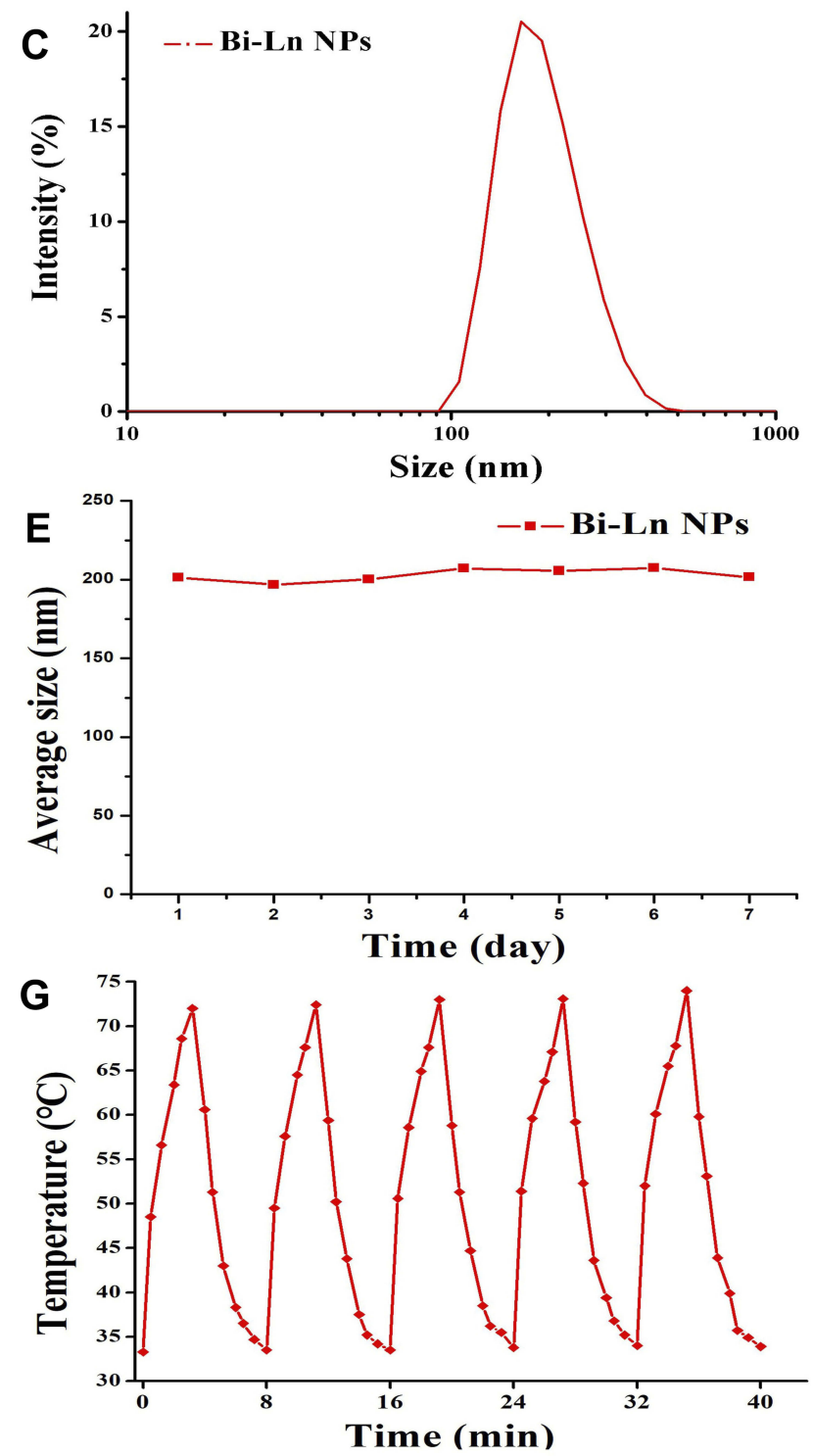

Figure I (A) TEM images of Bi NPs. (B) TEM images of Bi-Ln NPs. (C) Size distribution of Bi-Ln NPs determined by DLS. (D) UV-vis-NIR absorption spectra of Bi-Ln NPs. (E) Stability of Bi-Ln NPs during incubation in PBS for 7 days. (F) XRD spectra of Bi-Ln NPs. (G) Temperature variation of Bi-Ln NPs solution with 5 cycles of laser on/off $\left(808 \mathrm{~nm}, 1.0 \mathrm{~W} / \mathrm{cm}^{2}\right)$.

is mainly based on the enhanced permeability and retention (EPR) effect, enabling the extravasation and retention of macromolecules and nanocarriers at pathological sites. However, most nanoparticles are taken up by the Kupffer cells of the reticuloendothelial system after antitumor agents enter into the blood stream, ${ }^{40}$ only a small fraction of NPs $(\sim 0.7 \%$ injected dose) overcomes the transport and biological barriers and interacts with tumor tissue, a figure that can be further reduced for liver tumors due to their nonspecific uptake. ${ }^{41,42}$ Various strategies have been used to improve the delivery and tumor-specific targeting in PTT, including the modification of tumor-targeting ligands on the surface of PTAs. ${ }^{43}$ Moreover, biomimetic cancer cell membrane-coated nanoparticles have been developed to realize tumor targeting. ${ }^{44,45}$ However, limited tumor accumulation and low photothermal efficiency could restrict their further application. Therefore, new effective delivery strategies are still urgently needed for the increasing accumulation of nanomaterials in PTT.

Transarterial administration (TA) is a minimally invasive interventional clinical technique. ${ }^{46}$ Nanomaterials can be directly delivered into tumor sites through the artery that feeds the tumor. Current studies show that transcatheter intra-arterial infusion can enhance the accumulation of nanomaterials in tumors. ${ }^{47,48}$ Tian et $\mathrm{al}^{47}$ compared the tumor uptake of hollow gold nanospheres after intravenous 

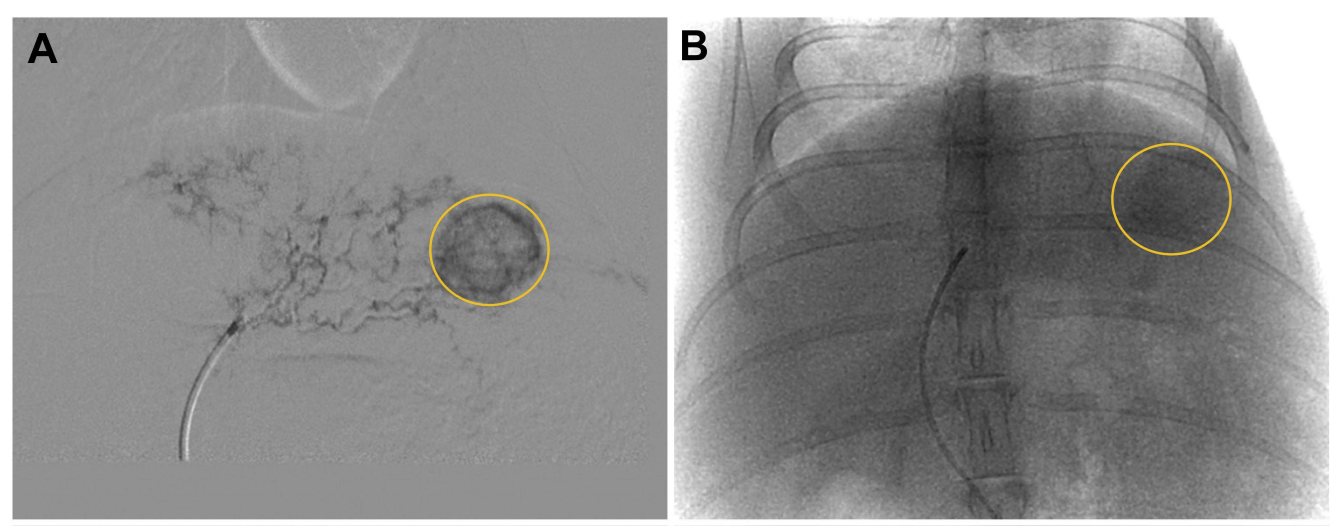

\section{IA Bi-Ln NPs}
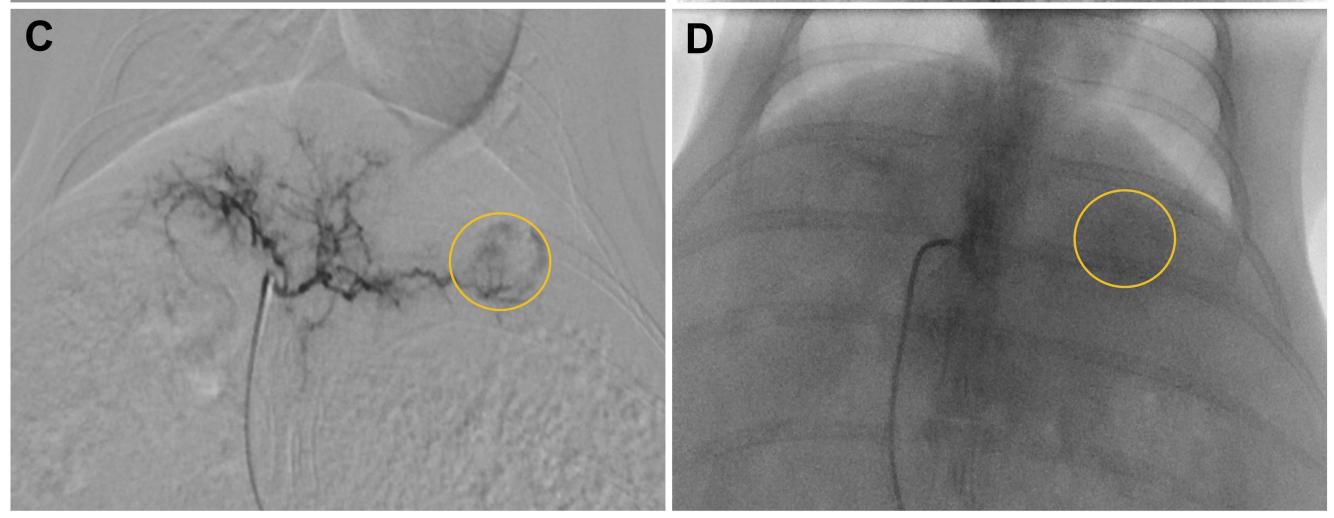

\section{IA PBS}

Figure 2 (A) Hepatic arteriogram before intra-arterial infusion of Bi-Ln NPs. (B) X-ray fluoroscopic image after intra-arterial infusion of Bi-Ln NPs. (C) Hepatic arteriogram before intra-arterial infusion of PBS. (D) X-ray fluoroscopic image after intra-arterial infusion of PBS.

A

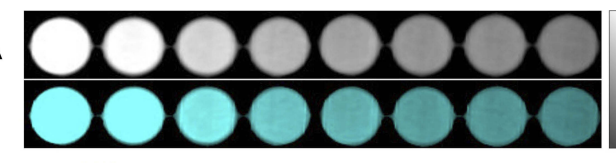

B
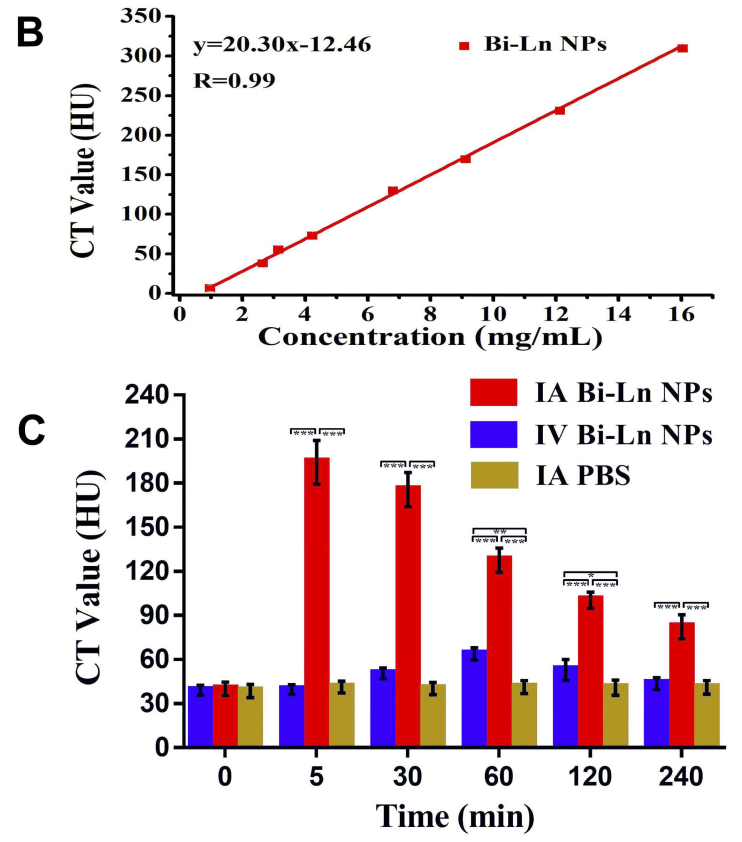

High $\mathrm{D}$

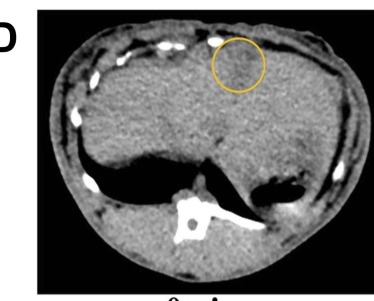

$0 \mathrm{~min}$

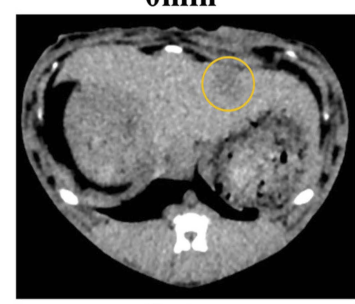

0min

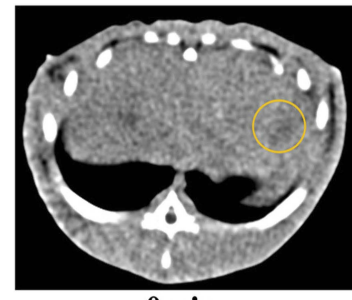

0min

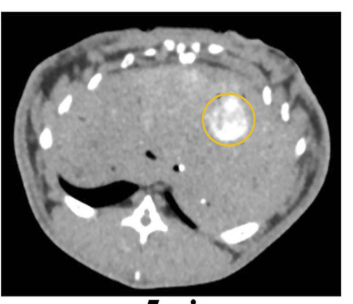

IA Bi-Ln NPs

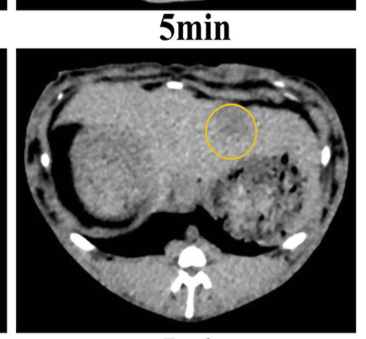

IA PBS

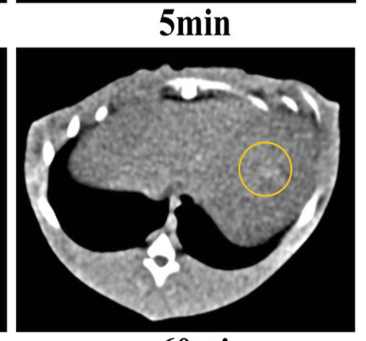

$60 \mathrm{~min}$

Figure 3 (A) In vitro CT images and the corresponding pseudocolor images of Bi-Ln NPs at different concentrations (I6.05, I2.13, 9.13, 6.8I, 4.24, 3.I5, 2.66, 0.98 mg/mL) and (B) corresponding CT values (HU). (C) CT values (HU) of tumor at different times ( 0 mins, 5 mins, 30 mins, I hr, 2 hrs, and 4 hrs) after injection in three groups (IA Bi-Ln NPs, IV Bi-Ln NPs, and IA PBS) ( ${ }^{* P}<0.05,{ }^{*} \mathrm{P}<0.0 \mathrm{I}$, $\left.* * \mathrm{P}<0.00 \mathrm{I}\right)$. (D) CT images of the tumor at 5 mins after intra-arterial infusion of Bi-Ln NPs and PBS, I hr after intravenous infusion of Bi-Ln NPs. 
and intra-arterial injection in a rabbit model and found that the intra-arterial injection group had higher ratios of tumor-to-normal liver uptake than the other groups. In this study, compared to the intravenous infusion group, the CT values of tumors increased significantly after intraarterial infusion of Bi-Ln NPs. This phenomenon indicated that the concentration of Bi-Ln NPs increased significantly at the tumor site. Therefore, via the intra-arterial infusion technique, the concentrations of nanomaterials in tumors improved significantly, which is critical for subsequent PTT treatment.

\section{In vitro and in vivo Photothermal Conversion of $\mathrm{Bi}-\mathrm{Ln}$ NPs}

Due to the high absorption in NIR (700-900 nm), Bi-Ln NPs may act as an efficient agent for photothermal conversion. For measuring in vitro photothermal properties, as depicted in Figure 4A, the temperature of the solution increased with the concentration of Bi-Ln NPs and the irradiation time. After $1 \mathrm{~min}$ of irradiation, the temperature of Bi-Ln NPs with a concentration of $2 \mathrm{mg} / \mathrm{mL}$ increased from $30.1^{\circ} \mathrm{C}$ to $63^{\circ} \mathrm{C}$. After irradiation for $3 \mathrm{mins}$, the solution temperature increased to $74^{\circ} \mathrm{C}$, demonstrating a high photothermal conversion efficiency of Bi-Ln NPs.

For in vivo photothermal conversion, tumor-bearing rabbits in groups $\mathrm{A}$ and $\mathrm{B}$ received $\mathrm{Bi}-\mathrm{Ln}$ NPs by intraarterial infusion and intravenous infusion, respectively. Meanwhile, rabbits in group $\mathrm{C}$ received $\mathrm{PBS}$ by intraarterial infusion. After the infusion of Bi-Ln NPs or PBS, the IPTT was conducted, and 5 mins after injection in groups $\mathrm{A}$ and $\mathrm{C}$ and $1 \mathrm{hr}$ after injection in group $\mathrm{B}$, the tumors received NIR laser treatment $\left(808 \mathrm{~nm}, 1.0 \mathrm{~W} / \mathrm{cm}^{2}\right.$, 3 mins). As shown in Figure 4B and C, tumor temperature changed negligibly in group $\mathrm{C}$ and increased slightly from $36.9^{\circ} \mathrm{C}$ to $47.4^{\circ} \mathrm{C}$ in group $\mathrm{B}$. Conversely, in group $\mathrm{A}$, the tumor temperature increased from $36.7^{\circ} \mathrm{C}$ to $60.2^{\circ} \mathrm{C}$ after irradiation for 3 mins, which can efficiently induce cancer cell protein inactivation and death.
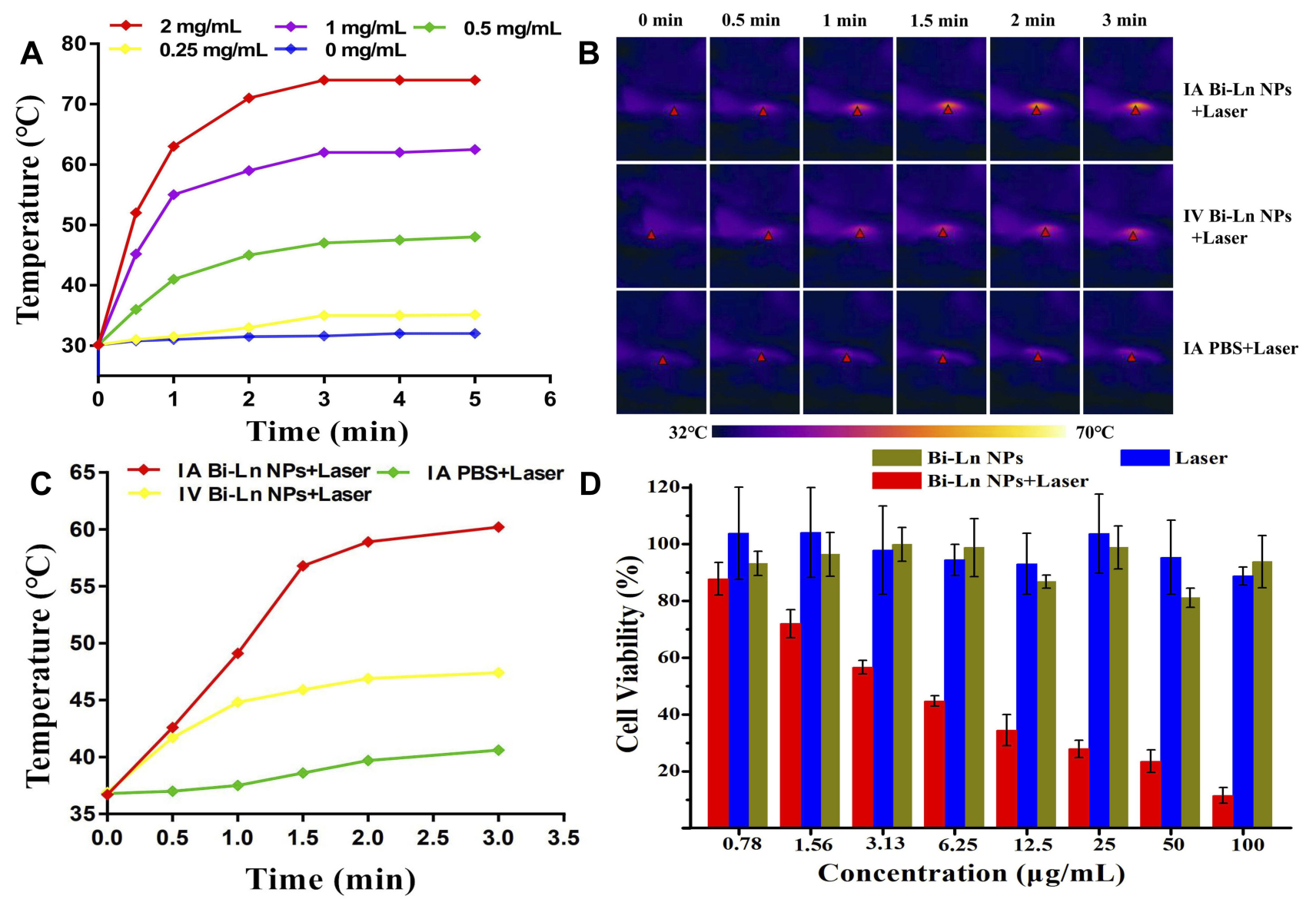

Figure 4 (A) The change curves of temperature with different concentration $(0,0.25,0.5, I, 2 \mathrm{mg} / \mathrm{mL})$ of Bi-Ln NPs under NIR laser $\left(808 \mathrm{~nm}\right.$, I.0 W/cm $\left.{ }^{2}\right)$. (B) Thermal images and (C) tumor area temperature of rabbit VX2 hepatic Carcinoma under 808-nm laser irradiation $\left(1.0 \mathrm{~W} / \mathrm{cm}^{2}\right)$ after infusion of Bi-Ln NPs or PBS in different group. (D) Cell viability of HepG 2 cells with or without $808-\mathrm{nm}$ laser irradiation ( $\mathrm{W} / \mathrm{cm}^{2}, 3$ mins). 
PTT depends on heat generation upon exposure of tumor cells to NIR. ${ }^{49}$ PTT agents absorb light energy, which causes electron excitation and subsequent nonradiative relaxation, which in turn results in heat generation in the medium surrounding the PTAs. ${ }^{50}$ Therefore, the photothermal conversion of PTAs and the light penetration depth of NIR are two significant factors in PTT. In this study, the in vitro photothermal results showed that Bi-Ln NPs exhibited a high photothermal conversion efficiency, consistent with previous studies. $^{22,25}$ IPTT could access the deep abdominal cavity for localized treatment, reducing the laser power attenuation caused by the percutaneous irradiation of traditional PTT. ${ }^{51}$ We conducted IPTT in hepatic VX2 carcinoma, and the increase in the tumor temperature due to the significant improvement in Bi-Ln NPs concentration in tumor tissue and the high photothermal conversion efficiency of Bi-Ln NPs was evident. In addition, minimally invasive IPTT could protect normal liver tissues around the tumor from being damaged by high temperature.

\section{In vitro Photothermal Cytotoxicity of Bi-Ln NPs}

Based on a previous study, Bi-Ln NPs can effectively convert NIR energy to thermal energy. The dark toxicity and phototoxicity were calculated by the MTT method, the results showed that Bi-Ln NPs exhibited negligible dark toxicity, demonstrating low cytotoxicity and striking biocompatibility and can be used for further biomedical applications. As shown in Figure 4D, the growth of HepG 2 cells was significantly inhibited after irradiation with NIR $\left(808 \mathrm{~nm}, 1.0 \mathrm{~W} / \mathrm{cm}^{2}\right)$ for $3 \mathrm{~min}$. This result demonstrated that Bi-Ln NPs possessed good biocompatibility and could effectively induce cell death under irradiation for PTT.

\section{In vivo IPTT}

To demonstrate the IPPT effect, we administered Bi-Ln NPs to tumor-bearing rabbits by intra-arterial infusion and intravenous infusion, respectively. Then, the IPTT efficacy was monitored by MRI. All VX2 hepatic carcinomas appeared homogeneous and hyperintense on T2WI prior to treatment. However, VX2 hepatic carcinoma showed inhomogeneity after treatment (Figure 5A). The tumor growth ratios (V/V0) and the TIR were calculated. As shown in Figure 5B and C, 7 days after treatment, compared to groups $\mathrm{C}$ and $\mathrm{D}$, the growth ratios of tumors (V/V0) in groups $\mathrm{A}$ and $\mathrm{B}$ were decreased, with group A decreasing more significantly. Compared to group $\mathrm{C}$, the tumor volumes in groups A and B showed different levels of inhibition. At the same

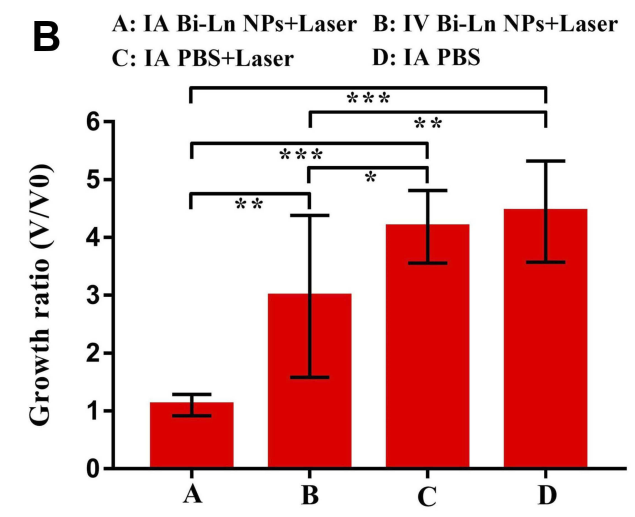

C A: IA Bi-Ln NPs+Laser B: IV Bi-Ln NPs+Laser C C:IA PBS+Laser

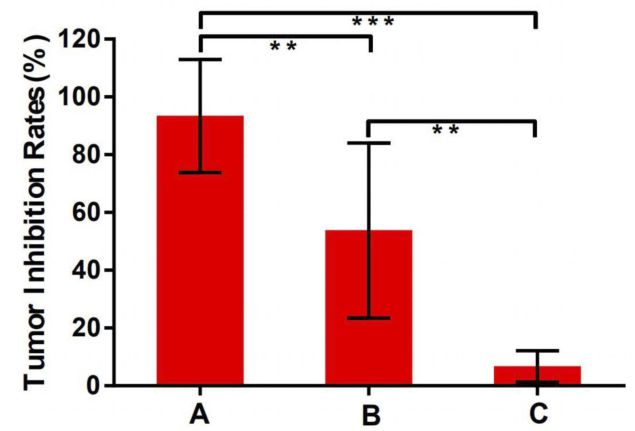

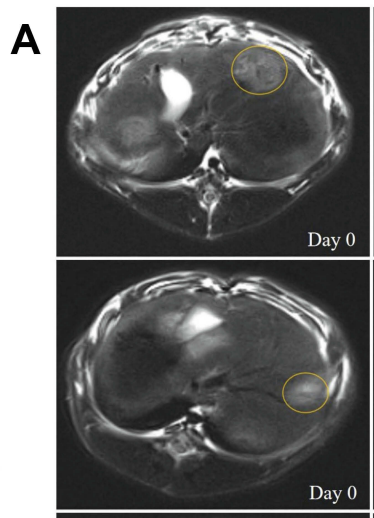

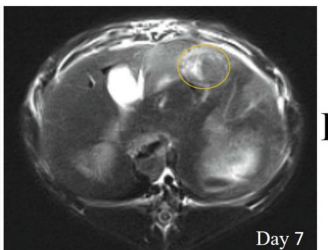

IA Bi-Ln NPs+Laser

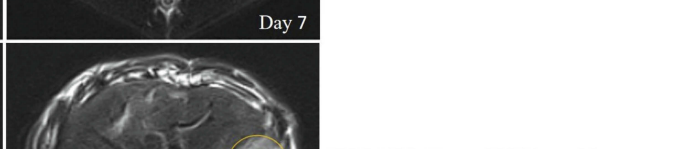

IV Bi-Ln NPs+Laser
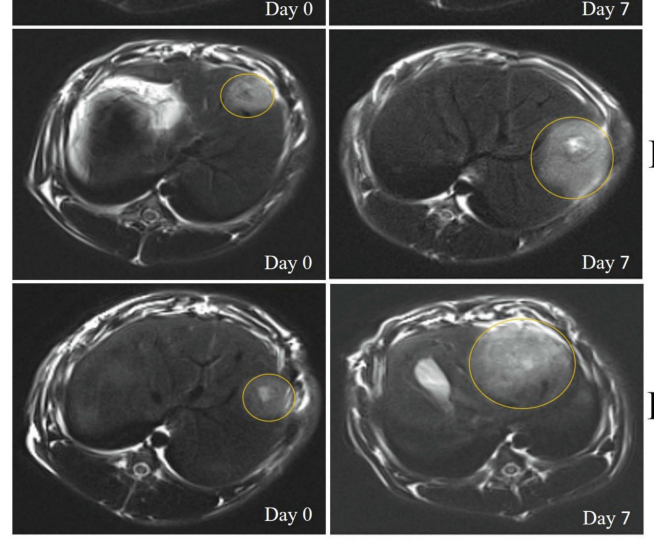

Figure 5 (A) T2-weighted images of tumor at day 0 and day 7 in different group. (B) Tumor growth ratios (V/V0) and $(\mathbf{C})$ TIR with different treatments $(* \mathrm{P}<0.05$, **P<0.0I, $* * * \mathrm{P}<0.00 \mathrm{I})$. 
time, the tumor growth ratios and the TIR between groups $\mathrm{A}$ and $\mathrm{B}$ showed significant differences. The tumor growth ratios in groups A and B were $1.1 \pm 0.19$ and $2.98 \pm 1.4$, and the TIRs were $93.38 \pm 19.57 \%$ and $53.78 \pm 30.28 \%$, respectively. It was evident that the intra-arterial infusion with the NIR laser treatment group showed higher inhibition of tumor growth.

\section{Histological and Immunohistochemical Assays}

As depicted in Figure 6A, H\&E staining showed small amounts of necrosis in groups $\mathrm{C}$ and $\mathrm{D}$. In contrast to group B, group A showed a large amount of nuclear shrinkage and heavy necrosis (necrosis rate was $83.12 \pm 8.02 \%$ ) (Figure 6B). TUNEL assay showed that apoptotic cell numbers increased in both groups A and B (Figure 6A), but group A displayed a significantly higher apoptosis rate (43.26 \pm $10.65 \%)$ than group B $(18.86 \pm 6.26 \%)$ (Figure 6C). The results of $H \& E$ and TUNEL assays demonstrated that the new method possessed more significant anticancer efficacy than the other groups.
PTT has attracted tremendous attention, as it is repeatable, highly spatiotemporal and effectively therapeutic. IPTT can deliver enough NIR directly to the tumor site through optical fiber, avoiding the limited light penetration of NIR. Zhang et al developed an interventional photothermal-brachytherapy (IPT-BT) strategy to fight deep pancreatic cancer, and the results showed that a high TIR of $96.6 \%$ is achieved in SW1990 orthotopic pancreatic tumor-bearing mice by HGN-mediated IPT-BT synergistic therapy. ${ }^{52}$ To improve the treatment effect of VX2 hepatic carcinoma, transcatheter intra-arterial infusion combined with IPTT was applied in our study. Furthermore, Bi-Ln NPs with high photothermal conversion efficiency were employed as PTAs. The results show a higher TIR, a higher tumor necrosis rate and a higher apoptosis rate, demonstrating the significant anticancer efficacy of intraarterial infusion combined with IPTT.

\section{Conclusion}

In summary, the water-dispersed Bi-Ln NPs in this study were synthesized using a simple emulsification method. Bi-Ln NPs exhibited excellent biocompatibility, a good

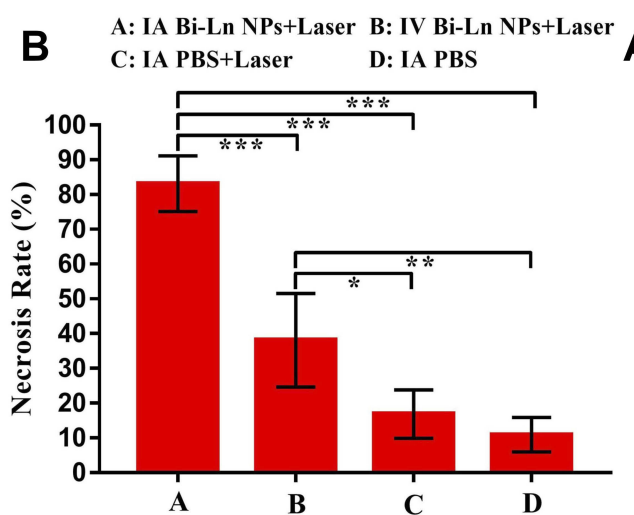

C

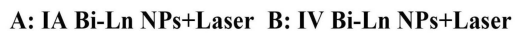

C: IA PBS+Laser D: IA PBS

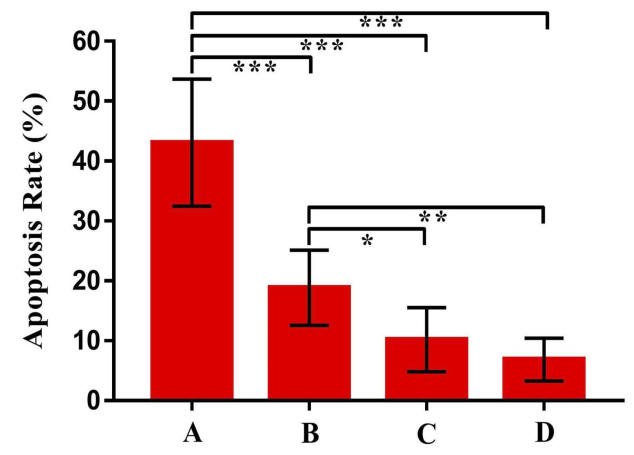

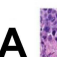

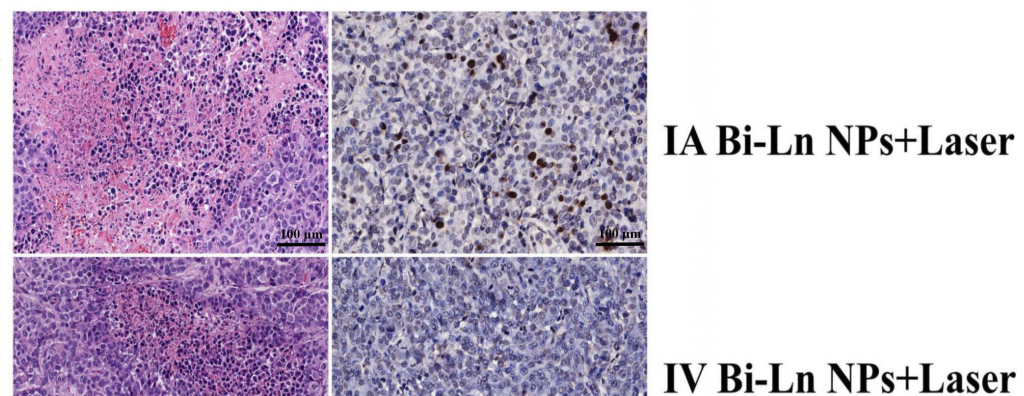

IV Bi-Ln NPs+Laser

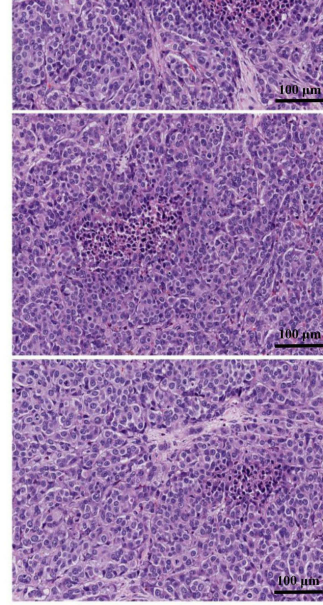

H\&E
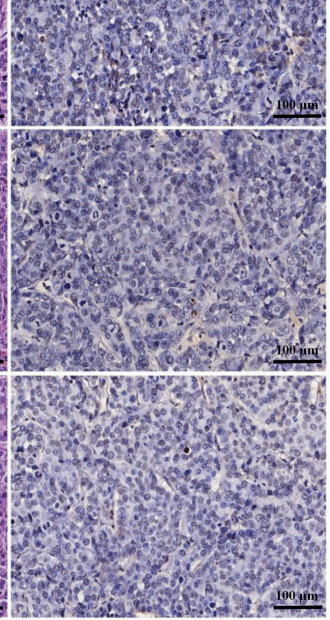

TUNEL

Figure 6 (A) H\&E staining and TUNEL of the tumor tissues collected from different groups after the last MRI test at day 7. (B) The necrosis rate and (C) apoptosis rate of tumor after different treatments $(* \mathrm{P}<0.05, * * \mathrm{P}<0.01$, $* * * \mathrm{P}<0.00 \mathrm{I})$. 
X-ray attenuation coefficient and a higher photothermal conversion efficiency. Intra-arterial infusion could efficiently increase the concentration of Bi-Ln NPs in tumors. Moreover, minimally invasive IPTT could be utilized to treat VX2 hepatic carcinoma and obtained excellent therapeutic effects. The feasibility and effectiveness results have shown the potential of this method and recommend transcatheter intra-arterial infusion Bi-Ln NPs combined with IPTT as a promising approach in cancer therapy.

\section{Acknowledgments}

The authors are deeply grateful to Dr Kai Deng and Dr Bo $\mathrm{Wu}$ for providing assistance to the synthesis of Bi-Ln NPs in this study. This work was supported by the Fundamental Research Funds for the Central Universities (81771819, 81571734), and Supported by Zhongnan Hospital of Wuhan University Science, Technology and Innovation Seed Fund (cxpy2017048, cxpy20160057).

\section{Author Contributions}

All authors contributed to data analysis, drafting and revising the article, gave final approval of the version to be published, and agree to be accountable for all aspects of the work.

\section{Disclosure}

The authors report no conflicts of interest in this work.

\section{References}

1. Wong MC, Jiang JY, Goggins WB, et al. International incidence and mortality trends of liver cancer: a global profile. Sci Rep. 2017;7:45846. doi:10.1038/srep45846

2. Kakushadze Z, Raghubanshi R, Yu WL. Estimating cost savings from early cancer diagnosis. Data. 2017;2(3):30. doi:10.3390/data2030030

3. Tsuchiya N, Sawada Y, Endo I, Saito K, Uemura Y, Nakatsura T. Biomarkers for the early diagnosis of hepatocellular carcinoma. World J Gastroenterol. 2015;21(37):10573-10583. doi:10.3748/wjg. v21.i37.10573

4. Farinati F, Sergio A, Baldan A, et al. Early and very early hepatocellular carcinoma: when and how much do staging and choice of treatment really matter? A multi-center study. BMC Cancer. 2009;9:33. doi:10.1186/1471-2407-9-33

5. Shrimal A, Prasanth M, Kulkarni AV. Interventional radiological treatment of hepatocellular carcinoma: an update. Indian J Surg. 2012;74 (1):91-99. doi:10.1007/s12262-011-0377-4

6. Jarnagin W, Chapman WC, Curley S, et al. Surgical treatment of hepatocellular carcinoma: expert consensus statement. HPB (Oxford). 2010;12(5):302-310. doi:10.1111/j.1477-2574.2010.00182.x

7. Mazzaferro V, Regalia E, Doci R, et al. Liver transplantation for the treatment of small hepatocellular carcinomas in patients with cirrhosis. New Engl J Med. 1996;334(11):693-699. doi:10.1056/NEJM19960 3143341104

8. Clavien PA, Lesurtel M, Bossuyt PM, et al. Recommendations for liver transplantation for hepatocellular carcinoma: an international consensus conference report. Lancet Oncol. 2012;13(1):E11-E22. doi:10.1016/S1470-2045(11)70175-9
9. Pomfret EA, Washburn K, Wald C, et al. Report of a national conference on liver allocation in patients with hepatocellular carcinoma in the United States. Liver Transpl. 2010;16(3):262-278. doi:10.1002/lt.v16:3

10. Llovet JM, Schwartz M, Mazzaferro V. Resection and liver transplantation for hepatocellular carcinoma. Semin Liver Dis. 2005;25 (2):181-200. doi:10.1055/s-2005-871198

11. Sangiovanni A, Colombo M. Treatment of hepatocellular carcinoma: beyond international guidelines. Liver Int. 2016;36(Sl):124-129. doi:10.1111/liv.2016.36.issue-S1

12. Poon RT, Fan ST, Tsang FH, Wong J. Locoregional therapies for hepatocellular carcinoma: a critical review from the surgeon's perspective. Ann Surg. 2002;235(4):466-486. doi:10.1097/00000658200204000-00004

13. Brown KT, Nevins AB, Getrajdman GI, et al. Particle embolization for hepatocellular carcinoma. $J$ Vasc Interv Radiol. 1998;9 (5):822-828. doi:10.1016/S1051-0443(98)70398-7

14. Covey AM, Maluccio MA, Schubert J, et al. Particle embolization of recurrent hepatocellular carcinoma after hepatectomy. Cancer. 2006;106(10):2181-2189. doi:10.1002/(ISSN)1097-0142

15. Camma C, Schepis F, Orlando A, et al. Transarterial chemoembolization for unresectable hepatocellular carcinoma: meta-analysis of randomized controlled trials. Radiology. 2002;224(1):47-54. doi:10.1148/radiol.224 1011262

16. Marelli L, Stigliano R, Triantos C, et al. Transarterial therapy for hepatocellular carcinoma: which technique is more effective? A systematic review of cohort and randomized studies. Cardiovasc Intervent Radiol. 2007;30(1):6-25. doi:10.1007/s00270-006-0062-3

17. Zou LL, Wang H, He B, et al. Current approaches of photothermal therapy in treating cancer metastasis with nanotherapeutics. Theranostics. 2016;6 (6):762-772. doi:10.7150/thno.14988

18. Zhu XJ, Feng W, Chang J, et al. Temperature-feedback upconversion nanocomposite for accurate photothermal therapy at facile temperature. Nat Commun. 2016;7:10437. doi:10.1038/ncomms10437

19. Xing RT, Liu K, Jiao TF, et al. An injectable self- assembling collagen-gold hybrid hydrogel for combinatorial antitumor photothermal/photodynamic therapy. Adv Mat. 2016;28(19):3669-3676. doi:10.1002/adma.201600284

20. Wei B, Zhang XJ, Zhang C, et al. Facile synthesis of uniform-sized bismuth nanoparticles for ct visualization of gastrointestinal tract in vivo. ACS Appl Mater Interfaces. 2016;8(20):12720-12726. doi:10.10 21/acsami.6b03640

21. Li A, Li X, Yu XJ, et al. Synergistic thermoradiotherapy based on PEGylated $\mathrm{Cu}_{3} \mathrm{BiS}_{3}$ ternary semiconductor nanorods with strong absorption in the second near-infrared window. Biomaterials. 2017;112:164-175. doi:10.1016/j.biomaterials.2016.10.024

22. Zha ZB, Deng ZJ, Li YY, et al. Biocompatible polypyrrole nanoparticles as a novel organic photoacoustic contrast agent for deep tissue imaging. Nanoscale. 2013;5(10):4462-4467. doi:10.1039/c3nr00627a

23. Kelong A, Yanlan L, Jianhua L, et al. Large-scale synthesis of $\mathrm{Bi}_{2} \mathrm{~S}_{3}$ nanodots as a contrast agent for in vivo x-ray computed tomography imaging. Adv Mater. 2011;23(42):4886-4891. doi:10. 1002/adma.201103289

24. Bogusz K, Tehei M, Stewart C. Synthesis of potential theranostic system consisting of methotrexate-immobilized (3-aminopropyl) trimethoxysilane coated alpha- $\mathrm{Bi}_{2} \mathrm{O}_{3}$ nanoparticles for cancer treatment. RSC Adv. 2014;4(46):24412-24419. doi:10.1039/c4ra02160f

25. Song G, Liang C, Gong H, et al. Core-shell mnse@ $\mathrm{Bi}_{2} \mathrm{Se}_{3}$ fabricated via a cation exchange method as novel nanotheranostics for multimodal imaging and synergistic thermoradiotherapy. Adv Mater. 2015;27(40):6110-6117. doi:10.1002/adma.201503006

26. Li Z, Hu Y, Chang M, et al. Highly porous PEGylated $\mathrm{Bi}_{2} \mathrm{~S}_{3}$ nano-urchins as a versatile platform for in vivo triple-modal imaging, photothermal therapy and drug delivery. Nanoscale. 2016;8:16005-16016. doi:10.1039/ C6NR03398A

27. $\mathrm{Yu}$ H, Chen M, Rice PM, et al. Dumbbell-like bifunctional $\mathrm{Au}-\mathrm{Fe}_{3} \mathrm{O}_{4}$ nanoparticles. Nano Lett. 2005;5(2):379-382. doi:10.1021/n1047955q 
28. Rabin O, Perez JM, Grimm J, Wojtkiewicz G, Weissleder R. An X-ray computed tomography imaging agent based on long-circulating bismuth sulphide nanoparticles. Nat Mater. 2006;5(2):118-122. doi:10.1038/ nmat 1571

29. Wu B, Lu ST, Yu H, et al. Gadolinium-chelate functionalized bismuth nanotheranostic agent for in vivo MRI/CT/PAI imaging-guided photothermal cancer therapy. Biomaterials. 2018;159:37-47. doi:10.1016/j. biomaterials.2017.12.022

30. Gai SL, Yang GX, Yang PP, et al. Recent advances in functional nanomaterials for light-triggered cancer therapy. Nano Today. 2018;19:146-187. doi:10.1016/j.nantod.2018.02.010

31. Jung HS, Verwilst P, Sharma A, Shin J, Sessler JL, Kim JS. Organic molecule- based photothermal agents: an expanding photothermal therapy universe. Chem Soc Rev. 2018;47(7):2280-2297. doi:10.1039/ C7CS00522A

32. Tsai MF, Chang SH, Cheng FY, et al. nanorod design as light-absorber in the first and second biological near-infrared windows for in vivo photothermal therapy. ACS Nano. 2013;7(6):5330-5342. doi:10.1021/ nn401187c

33. Liu HY, Liu TL, Wu XL, et al. Targeting gold nanoshells on silica nanorattles: a drug cocktail to fight breast tumors via a single irradiation with near-infrared laser light. Adv Mater. 2012;24(6):755-761. doi:10.1002/adma.201103343

34. Jeon MJ, Gordon AC, Larson AC, Chung JW, Kim YI, Kim DH. Transcatheter intra-arterial infusion of doxorubicin loaded porous magnetic nano-clusters with iodinated oil for the treatment of liver cancer Biomaterials. 2016;88:25-33. doi:10.1016/j.biomaterials.2016.02.021

35. Junjie L, Zhou M, Liu F, et al. Hepatocellular carcinoma: intra-arterial delivery of doxorubicin-loaded hollow gold nanospheres for photothermal- ablation chemoembolization therapy in rats. Radiology. 2016;281(2):427-435. doi:10.1148/radiol.2016152510

36. Zhang X, Dai F, Chen J, et al. Antitumor effect of curcumin liposome after transcatheter arterial embolization in VX2 rabbits. Cancer Biol Ther. 2019;20(5):642-652. doi:10.1080/15384047.2018.1550567

37. Sun H, Linfeng X, Fan T, et al. Targeted hyperthermia after selective embolization with ferromagnetic nanoparticles in a VX2 rabbit liver tumor model. Int J Nanomedicine. 2013;8:3795-3804. doi:10.2147/ IJN.S50373

38. Dong S, Tang Q, Long M, Guan J, Lu Y, Gaopeng L. The cooperative effect of p53 and $\mathrm{Rb}$ in local nanotherapy in a rabbit VX2 model of hepatocellular carcinoma. Int J Nanomedicine. 2013;8:3757-3768. doi:10.2147/IJN.S51353

39. Mikhail AS, Pritchard WF, Negussie AH, et al. Mapping drug dose distribution on CT images following transarterial chemoembolization with radiopaque drug-eluting beads in a rabbit tumor model. Radiology. 2018;289(2):396-404. doi:10.1148/radiol.2018172571
40. Jokerst JV, Lobovkina T, Zare RN, Gambhir SS. Nanoparticle PEGylation for imaging and therapy. Nanomedicine (Lond). 2011;6 (4):715-728. doi:10.2217/nnm.11.19

41. Wilhelm S, Tavares AJ, Dai Q, et al. Analysis of nanoparticle delivery to tumours. Nat Rev Mater. 2016;1(5):16014. doi:10.1038/ natrevmats.2016.14

42. MacParland SA, Tsoi KM, Ouyang B, et al. Phenotype determines nanoparticle uptake by human macrophages from liver and blood. ACS Nano. 2017;11(3):2428-2443. doi:10.1021/acsnano.6b06245

43. Zhao R, Han X, Li Y, et al. Photothermal effect enhanced cascade-targeting strategy for improved pancreatic cancer therapy by gold nanoshell@mesoporous silica nanorod. ACS Nano. 2017;11 (8):8103-8113. doi:10.1021/acsnano.7b02918

44. Chen Z, Zhao P, Luo Z, et al. Cancer cell membrane-biomimetic nanoparticles for homologous-targeting dual-modal imaging and photothermal therapy. ACS Nano. 2016;10(11):10049-10057. doi:10.1021/ acsnano.6b04695

45. Zhu JY, Zheng DW, Zhang MK, et al. Preferential cancer cell self-recognition and tumor self-targeting by coating nanoparticles with homotypic cancer cell membranes. Nano Lett. 2016;16 (9):5895-5901. doi:10.1021/acs.nanolett.6b02786

46. Wang YX, Zhu XM, Liang Q, Cheng CH, Wang W, Leung KC. In vivo chemoembolization and magnetic resonance imaging of liver tumors by using iron oxide nanoshell/doxorubicin/poly(vinyl alcohol) hybrid composites. Angew Chem Int Ed Engl. 2014;53(19):4812-4815. doi:10.1002/anie.201402144

47. Tian M, Lu W, Zhang R, et al. Tumor uptake of hollow gold nanospheres after intravenous and intra-arterial injection: PET/CT study in a rabbit VX2 liver cancer model. Mol Imaging Biol. 2013;15 (5):614-624. doi:10.1007/s11307-013-0635-x

48. Tengchuang M, Tan L, Shao H, et al. Direct to tumor: transarterial administration of gold nanoshells with enhanced targeting and photothermal therapy. $J$ Biomed Nanotechnol. 2017;13(7):795-804. doi:10.1166/jbn.2017.2398

49. Melancon MP, Zhou M, Li C. Cancer theranostics with near-infrared light- activatable multimodal nanoparticles. Acc Chem Res. 2011;44 (10):947-956. doi:10.1021/ar200022e

50. Camerin M, Rello S, Villanueva A, et al. Photothermal sensitisation as a novel therapeutic approach for tumours: studies at the cellular and animal level. Eur J Cancer. 2005;41(8):1203-1212. doi:10.1016/ j.ejca.2005.02.021

51. Hu YY, Chi CW, Wang SH, et al. A comparative study of clinical intervention and interventional photothermal therapy for Pancreatic Cancer. Adv Mater. 2017;29:1700448. doi:10.1002/adma.v29.33

52. Zhang F, Han X, Yanyan H, et al. Interventional photothermal therapy enhanced brachytherapy: a new strategy to fight deep pancreatic. Cancer Adv Sci. 2019;6:1801507.
International Journal of Nanomedicine

\section{Publish your work in this journal}

The International Journal of Nanomedicine is an international, peerreviewed journal focusing on the application of nanotechnology in diagnostics, therapeutics, and drug delivery systems throughout the biomedical field. This journal is indexed on PubMed Central, MedLine, CAS, SciSearch ${ }^{\mathbb{R}}$, Current Contents ${ }^{\mathbb{B}} /$ Clinical Medicine,
Journal Citation Reports/Science Edition, EMBase, Scopus and the Elsevier Bibliographic databases. The manuscript management system is completely online and includes a very quick and fair peer-review system, which is all easy to use. Visit http://www.dovepress.com/ testimonials.php to read real quotes from published authors. 